\title{
EL PERIODISMO RECONSTRUCTIVO COMO GÉNERO PERIODÍSTICO EN LA ERA DE LA POSMODERNIDAD DIGITAL
}

\section{RECONSTRUCTIVE JOURNALISM AS THE GENUINE JOURNALISTIC GENRE IN THE AGE OF DIGITAL POSMODERNISM}

\author{
Manuel Ruiz Rico'. Universidad de Sevilla. España. \\ manuel.ruiz@democmedia.com
}

\section{RESUMEN}

La ecología de la comunicación determina que las tecnologías de la comunicación determinan el perfil de cada sociedad determinada en que dichas tecnologías operan. De este modo y debido al auge de internet, la época actual de la Posmodernidad digital se caracteriza por los dispositivos móviles y las redes sociales, por un tipo de información hiperabundante y caótica, que circula a una velocidad hiperrápida. La consecuencia de esto es la suspensión de la credibilidad, la erosión de la experiencia de realidad y la presencia cada vez más abundante de desinformación y bulos. Así como cada tecnología configura cada tipo de sociedad, cada tipo de sociedad, según sus características, se identifica con un tipo periodismo y unos géneros predominantes. Esta investigación teórica se ha apoyado en la revisión bibliográfica pertinente sobre la temática mencionada. Debido a las características descritas de la Posmodernidad digital y puesto que la función elemental del periodismo es informar y testimoniar la realidad, en esta investigación se concluye que la etapa del periodismo interpretativo ha quedado superada y se propone una nueva, que se corresponde más adecuadamente con las características de la etapa actual. Esta nueva etapa sería la del periodismo reconstructivo. Los géneros genuinos de este tipo de periodismo y, por lo tanto, de la actualidad, son el reportaje en profundidad (o reconstructivo), la crónica y la verificación (o fact-checking).

PALABRAS CLAVE: periodismo reconstructivo; Posmodernidad digital; etapas del periodismo; realidad; verdad; desinformación; verificación.

\section{ABSTRACT}

The Ecology of Communication establishes that communication technologies determine the profile of any given society in which these technologies operate. Thus, the current era of digital Postmodernism is characterized, due to the rise of the Internet, mobile devices and social networks, by a type of information that is hyperabundant, chaotic and circulates at a hyper-fast speed. The immediate consequence of this is the suspension of credibility, the erosion of the experience of

\footnotetext{
${ }^{1}$ Manuel Ruiz Rico: doctor en Periodismo (2012) por la Universidad de Sevilla. Miembro del grupo de investigación DEMOC-MEDIA de la Facultad de Periodismo de dicha institución. Autor del ensayo Antonio Muñoz Molina. El Robinson en Nueva York (2012). El último artículo publicado ha sido: Truth as Literature: Ethics of Journalism and Reality in the Digital Society. Estudios sobre el Mensaje Periodístico 26(1), 307-315.
} 
reality and the increasing presence of disinformation and fake news. As each technology configures each type of society, each type of society, according to its characteristics, is identified with a predominant type of journalism and genres. This theoretical research has been built upon a thorough review of relevant bibliography. Due to the described characteristics of digital Postmodernism and since the elementary function of journalism is to inform and testify to reality, we suggest that the stage of interpretive journalism has been overcome and a new one is proposed, which corresponds more adequately with the characteristics of the current stage. We have called this new stage the stage of reconstructive journalism. The genuine genres of this type of journalism and, therefore, of the current stage, are the reportage-at-large, the chronicle and fact-checking.

KEYWORDS: reconstructive journalism; digital postmodernism; stages of journalism; reality; truth; fake news; fact-checking.

\section{Cómo citar el artículo:}

Ruiz Rico, E. (2020). El periodismo reconstructivo como género periodístico en la era de la posmodernidad digital. Revista de Ciencias de la Comunicación e Información, 25(1), 39-48.

doi: http://doi.org/10.35742/rcci.2020.25(1).39-48

\section{INTRODUCCIÓN}

Cualquier investigación que tenga como eje las relaciones entre la realidad y el lenguaje no puede obviar el contexto histórico en el que estas relaciones tienen lugar. En el caso de este trabajo, centrado en lo que hemos denominado la posmodernidad digital, hay un momento clave en esta dialéctica lenguaje-realidad. George Steiner (1989, pp. 117-118) la ha ubicado a finales del siglo XIX y principios del XX y la ha descrito de este modo:

La cuestión decisiva ha sido ésta: hasta la crisis del significado del significado que se inició a finales del siglo XIX, incluso el escepticismo más severo, la más subversiva de las antirretóricas, estaban comprometidos con el lenguaje. [...] Inconsciente consigo mismo en la medida en que ha buscado expresión, el escepticismo aceptó el contrato con el lenguaje. En mi opinión, este contrato se rompió por primera vez, en cualquier sentido minucioso y consecuente, en la conciencia especulativa y la cultura europea, centroeuropea y rusa entre las décadas de 1870 y 1930. Esta ruptura de la alianza entre la palabra y el mundo constituye una de las pocas revoluciones del espíritu verdaderamente genuinas en la historia de Occidente y define la propia Modernidad.

En su Romanticismo. Una odisea del espíritu alemán, esta crisis del lenguaje ha sido señalada por Rüdiger Safranski (2018, pp. 279-280) como una de las consecuencias propias del Romanticismo en su reacción contra la llustración. Es ésta una dialéctica que el propio filósofo alemán ubica en dicha obra como precursora de la Posmodernidad, esa etapa caracterizada, en palabras de Gianni Vattimo (1990, p. 43 ), como una sociedad transparente donde la noción de realidad se erosiona. 
Safranski menciona a su vez un momento definitivo en esa crisis de realidad derivada de la crisis del lenguaje; a saber, la frase de Nietzsche (2008, p. 222): "No hay hechos, sólo interpretaciones", lo que sentó las bases para el relativismo puro que tanto abordó el autor de La gaya ciencia y que caracteriza toda la Posmodernidad.

En lo que tiene que ver con el lenguaje, y por lo tanto con el periodismo (sobre todo con el escrito), esta crisis descrita por Steiner y Safranski convertiría al lenguaje en una herramienta de representación de la realidad $y$, por lo tanto, no en realidad misma. En tanto que un mecanismo mediador sería un elemento más o menos ficcionalizador incapaz de comunicar con objetividad o veracidad ni el mundo real ni los acontecimientos reales que en él acontecen.

El filósofo José Antonio Marina (1999, p. 146) lo ha explicado de este modo:

La lingüística padece con frecuencia un raro tipo de parálisis. Queda plasmada ante la prestancia de la estructura del lenguaje y se olvida de los actos que están haciéndola posible. Se mueve entonces en un terreno fantasmagórico, borgiano. Es como si decidiera vivir en un diccionario o una enciclopedia y pensara que allí está todo. [...] El significado de las palabras, decía Peirce, es una cadena infinita de palabras. Si esto es así, un diccionario es un infinito enredo de infinitas cadenas de infinitas palabras. No lo creo. En algún momento el diccionario tiene unos términos que sólo se pueden aclarar remitiendo no a una palabra sino a una experiencia.

Al separar el lenguaje de la realidad, la lingüística, presa del positivismo exacerbado propio de finales del siglo XIX y principios del XX, cayó en el error de considerar el lenguaje como un ente autónomo, como una fenómeno-en-sí y propugnó investigar el lenguaje como se investigan las células: como el objeto de una ciencia natural (y no cultural) en aras de la sacrosanta (y mal entendida) objetividad científica.

Por este motivo, argumenta Marina (1999, p. 51): "No es verdad que la palabra sea sólo el uso que hacemos de la palabra. [Se] elige porque va bien a sus propósitos, porque el concepto mancomunado que constituye su significado común ampara la ampliación que quiere introducir". O, dicho en palabras de otro filósofo (Searle, 1997, p. 175):

No hacemos mundos; hacemos descripciones que pueden o no casar con el mundo real. Pero todo esto implica que hay una realidad que existe independientemente de nuestro sistema de conceptos. Sin esa realidad, no hay nada a lo que aplicar el concepto.

El mundo existe, el lenguaje no es una ilusión sino una herramienta para contarlo y dar testimonio de los hechos que en él acontecen y, por lo tanto, el periodismo puede describirlo y narrarlo con objetividad y rigor (lo que no implica decir en su totalidad absoluta).

Sin embargo, la paradoja es, como sugiere Vattimo (1990, p. 43), que a medida que las herramientas para contarlo (las tecnologías de la comunicación) se han desarrollado y extendido, la noción misma de realidad se erosiona y la experiencia de esa realidad se debilita. 
La ecología de los medios de comunicación surgió como disciplina en 1971 de la mano de Neil Postman y Marshall McLuhan (Velásquez y otros, 2018). Esta nueva disciplina colocaba

la palabra medios al lado de la palabra ecología para comprender que no solamente interesan los medios, sino también las formas de interacción entre los medios y los seres humanos, que ofrecen a una cultura su carácter y que, podemos decir, la ayudan a preservar un equilibrio simbólico (Velásquez y otros, 2018).

De este modo, la ecología de los medios se centra no sólo en cómo las tecnologías de la información condicionan los propios medios de comunicación sino también y sobre todo en cómo la comunicación misma (producida por una determinada tecnología) influye y condiciona la forma de las sociedades en que esa comunicación tiene lugar.

La consecuencia del desarrollo de las tecnologías de la comunicación (telégrafo, teléfono, radio, televisión y, finalmente y desde los años 60 , internet) ha sido la globalización, la materialización del concepto de aldea global de McLuhan.

Esta globalización mediática ha supuesto una explosión informativa, tanto en cantidad como en velocidad. Vattimo (1990, p. 82) ve en esto la materialización del concepto de Hegel de Weltanschauungen, la exposición de las visiones del mundo. Por este motivo, señala el filósofo italiano (1990, p. 79), "los medios de comunicación hacen que cada vez sea menos posible la idea de una realidad".

En La sociedad transparente Vattimo realiza un análisis certero de la Posmodernidad. Sin embargo, es un libro escrito antes de la explosión digital, en 1989. En estos 31 años desde que apareciera el libro se ha producido la presencia definitiva y primordial de los medios de comunicación (nativos digitales o no) en internet así como el auge de las redes sociales y de la telefonía móvil inteligente. Como indica Scott (2005):

Aunque hubo fallidos esfuerzos para poner en internet textos en los 80 , la explosión real del periodismo digital sucedió con la llegada de los primeros buscadores comerciales, Netscape en 1994 y el Internet Explorer de Microsoft en 1995. Para 1996, la mayoría de los medios de información, escritos y audiovisuales, tenían presencia online.

Es a esta etapa actual de la globalización a la que denominamos en este trabajo Posmodernidad digital, caracterizada, como hemos descrito en otro trabajo (Ruiz Rico, 2020) por una información que circula en cantidades masivas, a una velocidad ultrarrápida y con una capacidad de diseminación y capilarización inédita hasta la fecha. Todo ello facilitado por una característica de la red que ningún otro medio de información había tenido con anterioridad: es al mismo tiempo canal de distribución y soporte (escrito y audiovisual al mismo tiempo, es decir, multimedia).

Una consecuencia inmediata de esta situación es la crisis de la credibilidad informativa. La otra cara de esto es el auge de las llamadas fake news, es decir, del bulo y la desinformación. 
Este es el escenario actual donde se produce la comunicación, la información y el ejercicio del periodismo. Y ello se enmarca en el contexto histórico-cultural descrito y que abarca, por un lado, unos 150 años (desde la crisis moderna del lenguaje) y, por otro, unas cuatro décadas (desde el auge de las tecnologías digitales de la información).

De este modo, siguiendo los preceptos descritos por la ecología de la comunicación, esta investigación analizará las repercusiones de esto en el ejercicio del periodismo. En cada etapa histórica ha predominado o sido genuino de ella un tipo concreto de periodismo y esto ha dependido, en gran medida, tanto de las características de esa etapa histórica como del tipo de tecnología propio de dicha etapa (lo que a su vez influía en el carácter y perfil de ese período determinado y de la sociedad de ese momento histórico).

En los epígrafes que siguen realizaremos un análisis de esas etapas históricas, la tecnología predominante y el tipo de periodismo característico en ellas para, por último, caracterizar y definir, una vez caracterizada esta etapa histórica y su tecnología, el típico de periodismo de la Posmodernidad digital, que hemos denominado periodismo de reconstrucción o periodismo reconstructivo.

\section{OBJETIVOS}

Como hemos defendido en el párrafo anterior, esta investigación se basa en los postulados de la ecología de la información para proponer una nueva etapa del periodismo caracterizada, a su vez, por un género predominante o cuanto menos genuino: el periodismo reconstructivo o de reconstrucción.

Para ello, se parte de la caracterización del marco histórico-cultural general y de carácter más global, a saber: primero, la crisis moderna del lenguaje y la realidad (finales del siglo XIX y principios del XX); segundo, la Posmodernidad como etapa de la erosión de la realidad debido a la aparición y auge de los medios de comunicación de masas a partir de los años 40 del siglo XX.

Esta erosión de la realidad alcanza su paroxismo especialmente en su fase actual (siglo XXI) con la explosión y primacía global de la comunicación digital multipunto (debido al auge de las redes sociales y la expansión de la telefonía y el internet móvil). Hemos denominado a esta subetapa posmoderna, para caracterizarla, la Posmodernidad digital.

Además de la propuesta de este nuevo tipo de periodismo, que denominamos periodismo reconstructivo, este trabajo realizará también una definición del mismo, lo que implica proponer a su vez qué función del periodismo y, por lo tanto, qué género periodístico es propio y genuino de esta etapa de la Posmodernidad digital, teniendo en cuenta sus características y el marco histórico-social determinado y genuino de este momento histórico. 


\section{METODOLOGÍA}

Para realizar esta propuesta y el análisis investigador que conlleva, este trabajo, en tanto que investigación teórica, se basa eminentemente en la bibliografía relacionada publicada al respecto.

\section{DISCUSIÓN}

Puesto que esta investigación trata de proponer una nueva etapa del periodismo e identificar en ella el género o los géneros periodísticos genuinos, se hace necesario partir de la clasificación tradicional de etapas el periodismo establecida por la bibliografía al respecto.

Ángel Benito realizó la ya clásica estratificación en el primer volumen de su Teoría general de la información, de 1973. Según Benito (1973, p. 71):

en líneas generales puede afirmarse que el periodismo posterior a 1850 supone una serie de conquistas de primera magnitud: la conquista de todas las capas de la sociedad, de casi todos los países de la tierra y de todos los temas [...] El periodismo de este siglo largo (1850-1973) puede dividirse en tres etapas bien definidas. Periodismo ideológico, periodismo informativo y periodismo de explicación. Esta clasificación, que responde a los últimos estudios de prensa comparada realizados en el mundo, se funda en la consideración de los fines que en cada uno de estos tres períodos se han propuesto los profesionales de la información.

Moreno Espinosa (2000) resume así la caracterización que hace Benito de esas tres etapas. El periodismo ideológico llegaría hasta el fin de la Primera Guerra Mundial. Se caracteriza

por ser un periodismo al servicio de ideas políticas y religiosas. Es un tipo de prensa con muy pocas informaciones y muchos comentarios, realizada sobre todo por literatos. En ella impera la opinión sobre la información y tienen importancia el artículo, el comentario y el ensayo (Moreno Espinosa, 2000).

En cuanto al periodismo informativo, aparece hacia 1870 y "coexiste durante cierto tiempo con el periodismo ideológico. Se centra más en la narración o relato de los hechos que en las ideas, como la etapa anterior. Tienen más importancia los géneros informativos: la noticia, la crónica y el reportaje" (Moreno Espinosa, 2000).

El periodismo de explicación aparece después de la Segunda Guerra Mundial. "Supone", abunda Moreno Espinosa (2000), "un reciclaje de las dos etapas anteriores, motivado, sobre todo, por la aparición de la radio y la televisión y las consecuencias sociales que de ello se derivan".

El periodismo de explicación, en consecuencia, añade Moreno Espinosa (2000):

Aborda los hechos en profundidad y utiliza equilibradamente los géneros básicos, relato y comentario, situándolos en una nueva perspectiva mediante la cual el lector encuentra los juicios de valor al lado de la narración de los hechos de forma inmediata. Pretende, por tanto, informar y crear opinión a la vez. 
En cuanto a la relación entre los géneros periodísticos y las etapas del periodismo, Moreno Espinosa (2001) aclara:

Los géneros periodísticos son el resultado de una lenta evolución histórica ligada a la evolución del mismo concepto de lo que se entiende por periodismo. A lo largo de la historia del periodismo cada uno de los géneros no ha tenido la importancia que en la actualidad nosotros le damos. La aparición histórica de los géneros periodísticos está estrechamente relacionada con las distintas etapas del periodismo en cuanto hecho cultural [la cursiva es nuestra].

De este modo, si la aparición de la radio y la televisión provocó el surgimiento del periodismo de explicación y explica la preeminencia en esta etapa de "los géneros básicos, relato y comentario" para "informar y crear opinión a la vez", como decía Moreno Espinosa (2000), con la llegada de la Posmodernidad digital, es decir, con el desarrollo y auge de internet en las últimas tres décadas ( $\mathrm{y}$ especialmente en los últimos diez años con la telefonía inteligente y las redes sociales), una nueva etapa del periodismo habría emergido: el periodismo reconstructivo.

Argumenta Vattimo (1990, p. 69) que la sociedad posmoderna es, per se, una "sociedad mediatizada [y en éstas] el principio de realidad parece consumirse y atenuarse". Y prosigue el filósofo italiano (1990, pp. 153-154): "La sociedad del espectáculo [...] no es sólo la sociedad de las apariencias manipuladas por el poder, es también una sociedad en la que la realidad se da con caracteres más débiles y fluidos".

Lo que Vattimo describe de manera preclara, en una obra publicada en 1989, es la sociedad del bulo, la etapa de las llamadas fake news. La primera consecuencia de esto es que, para el ciudadano medio, jamás ha sido más complicado distinguir lo verdadero de lo falso. Esto, a su vez, trae como consecuencia la incredulidad ante todo mensaje que se reciba, provenga de donde provenga, por la cantidad ingente de información que se recibe (que puede ser contradictoria) y puesto que también cada vez es más complejo identificar la fuente original de la información que se recibe.

El ciudadano medio recibe decenas y hasta cientos de inputs informativos diarios a través de todo tipo de canales: además de los clásicos (televisión, radio, prensa escrita y medios digitales), correos electrónicos, mensajes de WhatsApp, mensajes de Twitter, contenidos de Facebook, etcétera. Las dos consecuencias descritas (la creencia en el bulo y la suspensión de la credibilidad ante cualquier mensaje) son nefastas para el ejercicio del periodismo profesional, cuya finalidad básica y razón de ser es contar los hechos, testimoniar la realidad.

Si lo comunicado se pone en tela de juicio, desaparece la función básica del periodismo (vigilancia de los poderes, público -Estado- y privado -empresas-, hacer de altavoz de los desfavorecidos para denunciar determinadas injusticias, dar a conocer hechos o verdades que se quieren ocultas, contar historias reales de interés humano, informar de hechos de interés público, etc.).

La Posmodernidad digital conlleva el establecimiento de una realidad caótica debido a la cantidad masiva de información circulante, a una velocidad ultrarrápida y con una capilarización jamás vistas en la historia de la humanidad. 
En un artículo de 2009, Javier Marías describió de manera preclara esta sensación, desde las páginas mismas de un medio escrito (El País Semanal):

Nunca me había sucedido lo que me sucede a menudo últimamente: leo una información intentando enterarme de lo que ocurre en un lugar determinado, o de cómo está la situación de tal conflicto, o de cuáles van a ser los problemas del libro cuando se generalicen el e-book y similares, o de qué va a pasar con la fosa de García Lorca, y no lo consigo. En el mejor de los casos, me quedo como estaba, y en el peor, han aumentado mi ignorancia y mi confusión.

El periodismo reconstructivo, por lo tanto, surge como ese tipo de periodismo que tiene como principal función informar pero no tanto para dar una exclusiva o una primicia sino, nunca mejor dicho, para dar forma, es decir: alejarse del bombardeo incesante y veloz del curso de las noticias de cada segundo (en el que la noticia de un momento corrige o contradice la noticia dada sobre un mismo hecho segundos o minutos antes...) para abordar acontecimientos y contarlos en toda su complejidad, generalmente, una vez el acontecimiento en cuestión, o una parte sustancial del mismo, haya concluido.

Se trata de establecer una narrativa (expresión no por casualidad tan manida en los últimos años), de desbrozar, de lo que se ha ido contando, lo que finalmente es y lo que finalmente no se corresponde con la realidad.

En España, una obra iniciática en este sentido se publicó en forma de libro: la novela de no-ficción de Javier Cercas Anatomía de un instante, publicada en 2009. Cercas toma como tema central de este libro el Golpe de Estado del 23-F, un acontecimiento de envergadura alrededor del cual se han vertido multitud de bulos y sobre el que tantas teorías de la conspiración existen.

El libro exhibe un abundante trabajo de documentación (los sumarios del caso, multitud de entrevistas, de trabajo de hemeroteca, incluso la cinta completa de la grabación de TVE de la jornada del Congreso, etc.), que Cercas desgrana con un estilo narrativo clásico y el empleo de la primera persona al apelar a menudo a su memoria personal.

Este uso del yo narrativo lejos de mermar el carácter objetivo de la narración, ahonda en su veracidad: el narrador no es alguien que habla de oídas, es alguien que se ha documentado, ha entrevistado a decenas de personas, es un escritor reconocido que publica en una editorial reconocida y que apela a su memoria personal y lo cuenta en primera persona (apelando a su pathos) y cuando opina lo deja claro: "Eso es lo que yo creo que ocurrió", llega a decir en un momento la obra $(309,2009)$. ¿Qué puede haber más objetivo que eso?

Vemos la realidad a través de sus ojos pero, sin duda, en medio de esta época de realidad erosionada, necesitamos que el periodista entreviste, se documente, esté en los sitios y cuenta las cosas como éstas surgen ante sus ojos. Hay un relato, una narración, es cierto, puede haber un yo escrito en primera persona pero esto no va en detrimento de la objetividad de lo narrado sino que es un eficaz antídoto contra el bulo y contra los negadores de la realidad y los hechos. 


\section{CONCLUSIONES}

Hemos propuesto el ejemplo de Anatomía de un instante para mostrar el modus operandi y la pertinencia del periodismo de reconstrucción.

Por lo tanto, los géneros clave del periodismo reconstructivo serían el reportaje (si se quiere en profundidad), la crónica y la verificación o fact-checking, que cada vez ocupa mayor espacio en los medios de información.

Como hemos adelantado, el reportaje se centraría en abordar temas complejos y de envergadura y con multitud de aristas para crear un relato coherente y de información verificada sobre lo acontecido.

En cuanto a la crónica, su función sería fundamentalmente poner los ojos del periodista en un entorno de interés y sobre el que hay información confusa para que dé testimonio de qué está sucediendo, lo que hace de ella, en buena medida, un método de verificación. Uno de los escenarios más decisivos de esto es en el periodismo de guerra, donde los periodistas pueden testimoniar hechos decisivos difíciles de corroborar salvo que uno esté sobre el terreno, como si una ciudad ha sido liberada o si se han producido ataques con armas químicas sobre la población, por ejemplo.

Por último, la verificación o fact-checking es el cotejo de los datos o las afirmaciones realizadas con el fin de confirmar que se ajustan a la realidad o desmentirlas. Suele ser el género estrella durante las noches de debates electorales.

Las funciones y la finalidad de estos géneros periodísticos son claras: fijar la realidad, determinar qué es verdad y qué es falso en el seno de una era digital donde cada vez la realidad es más débil y aparece ante el ciudadano erosionada y esquiva, con las consecuencias devastadoras que esto puede tener para el funcionamiento adecuado de los sistemas democráticos.

En la época de la erosión de la realidad, del bulo y la desinformación de las redes sociales y de la débil experiencia de verdad, el periodismo reconstructivo emerge como una nueva etapa del periodismo y como antídoto contra la falsedad, la mentira y los negacionistas de los hechos de la realidad.

\section{REFERENCIAS}

Benito, Á. (1973). Teoría General de la Información: I. Introducción. Madrid: Biblioteca Universitaria Guadiana.

Cercas, J. (2009). Anatomía de un instante. Barcelona: Mondadori.

Marías, J. (8 de noviembre, 2009). Que no me entero. El País Semanal. https://elpais.com/diario/2009/11/08/eps/1257665223 850215.html

Marina, J. A. (1999). La selva del lenguaje. Introducción a un diccionario de los sentimientos. Barcelona: Anagrama. 
Moreno Espinosa, P. (2000). Los géneros periodísticos informativos en la actualidad internacional. Revista Ámbitos, 5, 169-190. Recuperado de https://cutt.ly/lyC5SFI

Nietzsche, F. (2008). Fragmentos póstumos. Volumen IV. Segunda edición. Madrid: Tecnos.

Ruiz Rico, M. (2000). Truth as Literature: Ethics of Journalism and Reality in the Digital Society. Estudios sobre el Mensaje Periodístico, 26(1), 307-315.

Safranski, R. (2018). Romanticismo. Una odisea del espíritu alemán. Barcelona: Tusquets.

Scott, A. (2005). A Contemporary History of Digital Journalism. Television \& New Media, (6), 89. Recuperado de http://tvn.sagepub.com/cgi/content/abstract/6/1/89

Searle, J. R. (1997). La construcción de la realidad social. Barcelona: Paidós.

Steiner, G. (1989). Presencias reales. Madrid: Destino.

Vattimo, G. (1990). La sociedad transparente. Barcelona: Paidós.

Velásquez, A., Renó, D. Beltrán, A. M., Maldonado, J. C., y Ortiz León, C. (2018). De los mass media a los medios sociales: reflexiones sobre la nueva ecología de los medios. Revista Latina de Comunicación Social, (73), 583-594. doi: http://www.revistalatinacs.org/073paper/1270/29es.html

\section{AUTOR:}

\section{Manuel Ruiz Rico}

Doctor en Periodismo (2012) por la Universidad de Sevilla. Miembro del grupo de investigación DEMOC-MEDIA de la Facultad de Periodismo de dicha institución. Autor del ensayo Antonio Muñoz Molina. El Robinson en Nueva York (2012). El último artículo publicado ha sido: Truth as Literature: Ethics of Journalism and Reality in the Digital Society. Estudios sobre el Mensaje Periodístico 26(1), 307-315. Otros artículos de investigación han sido publicados por revistas como Textual and Visual Media o la revista Ínsula. Miembro de la FAPE 27.831. Periodista con 20 años de experiencia en España y el extranjero (Etiopía, Panamá, Dinamarca, Bélgica y Estados Unidos). Desde septiembre de 2019 ejerce de corresponsal del diario Público en Washington.

manuel.ruiz@democmedia.com

Orcid ID: https://orcid.org/0000-0002-8540-3866 